\title{
Effect of toroidal field ripple on plasma rotation in JET
}

\author{
P.C. de Vries ${ }^{1}$, A. Salmi ${ }^{2}$, V. Parail ${ }^{1}$, C. Giroud ${ }^{1}$, Y. Andrew ${ }^{1}$, \\ T.M. Biewer ${ }^{3}$, K. Crombét ${ }^{4}$ I. Jenkins ${ }^{1}$, T. Johnson ${ }^{5}$, V. Kiptily ${ }^{1}$, \\ A. Loarte $^{6}$, J. Lönnroth ${ }^{2}$, A. Meigs ${ }^{1}$, N. Oyama ${ }^{7}$, R. Sartori ${ }^{6}$, \\ G. Saibene ${ }^{6}$, H. Urano ${ }^{7}$, K.-D. Zastrow ${ }^{1}$ and \\ JET EFDA Contributors ${ }^{\mathrm{a}}$ \\ JET-EFDA Culham Science Centre, OX14 3DB, Abingdon, UK \\ ${ }^{1}$ EURATOM/UKAEA Fusion Association, Culham Science Centre, OX14 3DB, \\ Abingdon, UK \\ ${ }^{2}$ Association Euratom-Tekes, Helsinki University of Technology, PO Box 4100, 02015 TKK, \\ Finland \\ ${ }^{3}$ Oak Ridge National Laboratory, Oak Ridge, TN 37831, USA \\ ${ }^{4}$ Department of Applied Physics, Ghent University, Ghent, Belgium \\ ${ }^{5}$ Association EURATOM - VR, Fusion Plasma Physics, EES, KTH, Stockholm, Sweden \\ ${ }^{6}$ EFDA Close Support Unit, c/o MPI für Plasmaphysik, 2 Boltzmannstrasse, \\ 85748 Garching, Germany \\ ${ }^{7}$ Japan Atomic Energy Agency, Naka, Ibaraki-ken 311-0193, Japan \\ E-mail: Peter.de.Vries@jet.uk
}

Received 31 August 2007, accepted for publication 26 November 2007

Published 23 January 2008

Online at stacks.iop.org/NF/48/035007

\begin{abstract}
Dedicated experiments on TF ripple effects on the performance of tokamak plasmas have been carried out at JET. The TF ripple was found to have a profound effect on the plasma rotation. The central Mach number, $M$, defined as the ratio of the rotation velocity and the thermal velocity, was found to drop as a function of TF ripple amplitude $(\delta)$ from an average value of $M=0.40-0.55$ for operations at the standard JET ripple of $\delta=0.08 \%$ to $M=0.25-0.40$ for $\delta=0.5 \%$ and $M=0.1-0.3$ for $\delta=1 \%$. TF ripple effects should be considered when estimating the plasma rotation in ITER. With standard co-current injection of neutral beam injection (NBI), plasmas were found to rotate in the co-current direction. However, for higher TF ripple amplitudes $(\delta \sim 1 \%)$ an area of counter rotation developed at the edge of the plasma, while the core kept its co-rotation. The edge counter rotation was found to depend, besides on the TF ripple amplitude, on the edge temperature. The observed reduction of toroidal plasma rotation with increasing TF ripple could partly be explained by TF ripple induced losses of energetic ions, injected by NBI. However, the calculated torque due to these losses was insufficient to explain the observed counter rotation and its scaling with edge parameters. It is suggested that additional TF ripple induced losses of thermal ions contribute to this effect.
\end{abstract}

PACS numbers: 52.55.Fa, 52.65.Cc, 52.50.Gj

(Some figures in this article are in colour only in the electronic version)

\section{Introduction}

Plasma rotation is thought to play an important role in the stability properties of tokamak plasmas. Furthermore, rotational shear could affect plasma turbulence and consequently has an impact on transport processes and the performance of tokamak plasmas. Hence it is relevant to accurately predict the rotation properties of ITER plasmas.

a See appendix of Watkins M.L. et al 2006 Proc. 21st Int. Conf. on Fusion Energy 2006 (Chengdu, China) (Vienna: IAEA).
Because of the finite number of toroidal field (TF) coils, a toroidal variation (ripple) of the main magnetic field exists in tokamaks. The TF ripple breaks the axisymmetry of the magnetic field and enhances particle losses, in particular energetic ions, such as alpha particles created in fusion reactions or those injected by the neutral beam (NB) system. These non-ambipolar ion losses are expected to have a nonnegligible effect on the plasma rotation. Ferrite material will be mounted between the ITER coils in order to reduce the ripple. Nevertheless, the estimated maximum TF ripple at the 
separatrix in ITER is in the order of $\delta \sim 0.5 \%$ while this value in JET is only $\delta \sim 0.08 \%$. The maximum ripple at the separatrix is mostly found at the outboard mid-plane. Here the TF ripple amplitude, $\delta$, is defined as the relative amplitude of the magnetic field variation: $\delta=\left(B_{\max }-B_{\min }\right) /\left(B_{\max }+B_{\min }\right)$. In order to extrapolate the rotation properties of present experiments to that of ITER plasmas, TF ripple effects should be understood.

Studies on TF ripple induced particle losses have been reported by several devices [1-3]. Previous experiments on JET reported a reduction of the NB driven plasma rotation with TF ripple [4]. In JT-60U significant plasma rotation in the counter-current direction was observed with near perpendicular neutral beam injection (NBI) in the presence of a TF ripple of $\delta \sim 1 \%$ [5]. However, the rotation characteristics of the JT-60U plasmas were altered after the TF ripple was reduced using ferrite inserts [6,7]. Up to now, no detailed, quantitative analysis of TF ripple effects on plasma rotation has been carried out.

At JET it is possible to vary the TF ripple amplitude by independently powering the odd and even-numbered TF coils. The imbalance current between the two sets of coils can be changed arbitrarily, increasing the TF ripple up to $\delta \sim 3 \%$. A series of experiments has been performed analysing the effects of TF ripple by increasing its value on a pulse-by-pulse basis from the standard JET value of $\delta \sim 0.08 \%$ up to $\delta \sim 1.5 \%$. The plasma rotation has been measured by means of charge exchange recombination spectroscopy (CXRS). The measured quantities are those of carbon ions and here it is assumed that the main plasma ions have equal temperature and velocities.

This paper presents the effects of an enhanced TF ripple on the plasma rotation. In the second section the scaling of JET plasma rotation with TF ripple is presented. The basic mechanisms via which TF ripple affects the plasma rotation are discussed and experimental examples are shown. The experimental observations are compared with modelling of TF ripple induced particle losses. By means of the orbit following Monte Carlo code ASCOT, the trajectories of energetic ions, for example those injected by the NB system and those accelerated by ICRH, can be accurately traced in the presence of TF ripple [8]. This enables the determination of the fraction of energetic particles lost due to the TF ripple. These calculations are discussed in section 3 and compared with detailed experimental analysis. Finally the observations are summarized and the implications for ITER are discussed in section 4 .

\section{TF ripple and plasma rotation}

Dedicated experiments were carried out in which the TF ripple in JET was artificially increased. These experiments were done with various plasma parameters. In order to compare rotation properties of different experiments, it is convenient to use the dimensionless thermal Mach number, defined as the ratio of the rotation velocity and the thermal or ion sound velocity [9]. In figure 1, the toroidal Mach numbers measured at the plasma centre $(R=3.1 \mathrm{~m}, \rho \sim 0)$ and edge $(R=3.8 \mathrm{~m}$, $\rho \sim 0.9)$ are shown as a function of TF ripple amplitude, where $R$ is the location in major radius and the $\rho$ co-ordinate is the

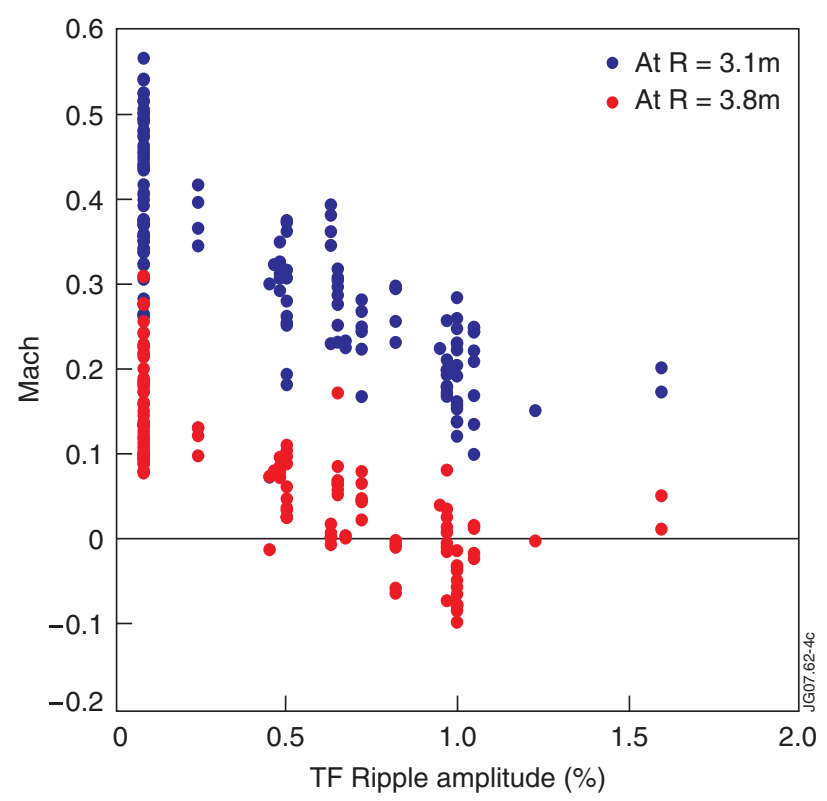

Figure 1. Mach numbers measured at the plasma centre $(R=3.1 \mathrm{~m}, \rho \sim 0)$ and edge $(R=3.8 \mathrm{~m}, \rho \sim 0.9)$ are shown asa function of TF ripple amplitude at the outer separatrix. The data have been taken over various scenarios and plasma conditions.

normalized square root of the poloidal flux. The central Mach number decreases from standard values of $M=0.40-0.55$ to $M=0.25-0.35$ for $\delta=0.5 \%$ and $M=0.1-0.3$ for $\delta=1 \%$. For these experiments the NBI system is the only external source of momentum.

A few data points around $\delta \sim 1.5 \%$ seem to break the trend. However, one should be aware that these discharges were all in the L-mode with low levels of NBI power $\left(\sim 3-4 \mathrm{MW}^{-1}\right)$. At this TF ripple amplitude, NBI operations was restricted due to the power large loss fraction and the resulting power loads on in-vessel components. JET operation at the predicted ITER TF ripple amplitude of $\delta=0.5 \%$ shows a significantly lower rotation compared with those at $\delta=0.08 \%$. For higher TF ripple amplitudes $(\delta \sim 1 \%)$ negative Mach numbers, i.e. counter rotation, is observed at the edge in various discharges while the core still rotates in the co-current rotation. The core and edge poloidal rotation velocity have been measured for plasmas with and without TF ripple. First results indicate that there is no significant effect of TF ripple on the core poloidal rotation. The influence of ripple on poloidal rotation in the core and edge region is currently under investigation.

The question that arises is, how does the TF ripple reduce toroidal plasma rotation? But firstly it is useful to recall the mechanisms of NBI momentum transfer to the bulk plasma. Because of the tangency of the neutral beams at JET, the particles carry toroidal momentum and the NBI system exerts a toroidal torque on the plasma which is directed in the co-current direction (see figure 2$)$. This torque, called NBI torque $\left(T_{\mathrm{NBI}}\right)$ in this paper, depends on the energy of the beam particles, particle flux and the tangency of the beams. There are three mechanisms explaining how this NBI torque acts on the plasma and the NBI momentum is transferred, which are described in detail in [10]. After ionization the injected NB particle can either become a passing particle or be trapped in a banana-orbit. 


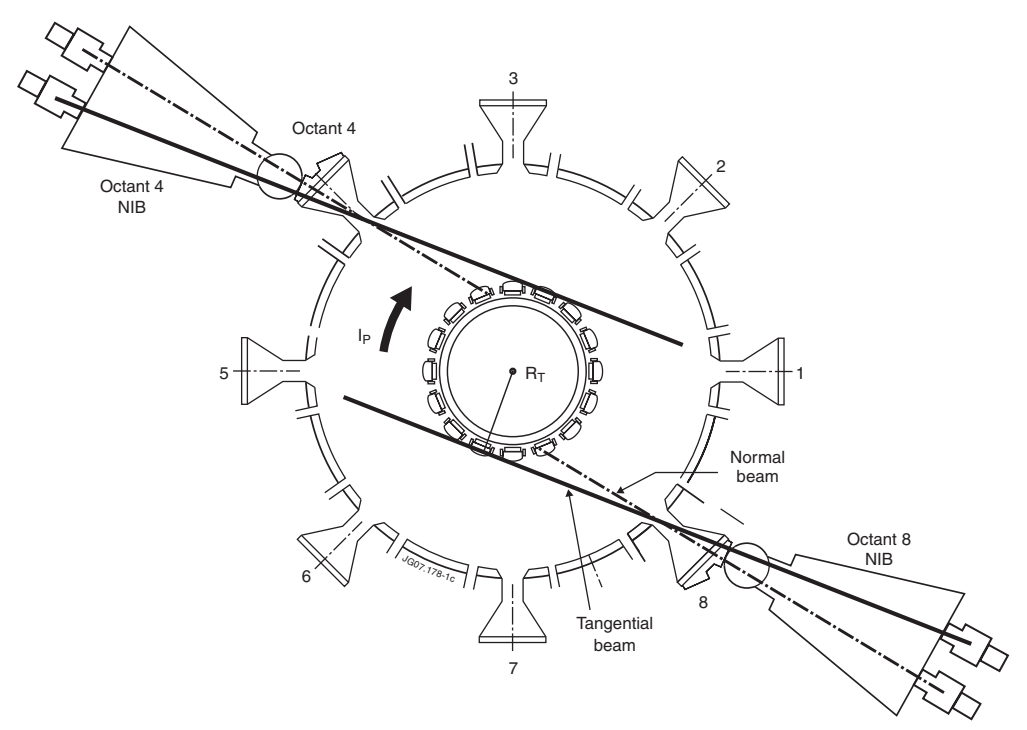

Figure 2. The location of two NB heating systems are shown on the top view of the JET torus. Both inject usually in the co-current direction. Each system consists of eight independent NB injectors, of which half are aligned with a tangency radius of $R_{\mathrm{T}}=1.31 \mathrm{~m}$ (the so-called normal injection) while the other half have a tangency radius of $R_{\mathrm{T}}=1.85 \mathrm{~m}$ (the so-called tangential injection), as indicated in the figure. The major radius of the JET torus is $R_{\mathrm{o}}=2.95 \mathrm{~m}$.

This depends on the position where the particle is deposited and the tangency of the NB. Passing particles transfer their momentum in two ways, either via collisions with the bulk plasma or by enhancement of the total angular momentum once the particles can be assumed thermalized. The characteristic transfer rate of these two mechanisms is in the order of the ion-ion collision time. The third mechanism is connected to those particles injected into banana-orbits. Since there is a radial separation of the position where the NB particle is ionized and its bounce averaged radial position, there is a radial current of fast ions. In order to maintain quasi-neutrality, there has to be a radial displacement current in the background plasma resulting in a $\boldsymbol{j} \times \boldsymbol{B}$ torque. This torque is transferred during one bounce time of the trapped particles and because of this fast time scale this torque component is often called the 'instantaneous' torque.

The presence of a TF ripple affects the energetic particles that are, for example, injected by the NB system in two ways. Firstly, particles can be trapped in the magnetic ripple. Being toroidally trapped, the field curvature causes these particles to drift out of the plasma. Secondly, the TF ripple alters the trajectory of particles trapped in banana-orbits and causes these to drift radially outwards [11]. Both losses have an effect on the plasma rotation. Toroidal rotation can be damped by friction between the circulating particles and those locally trapped in the TF ripple [12]. Furthermore, the TF ripple can be viewed as an external force that drives non-ambipolar radial current. The outward ion loss flow, due to, for example, banana-orbit diffusion, induces a radial return current, $j$, in order to preserve neutrality. This gives rise to a $\boldsymbol{j} \times \boldsymbol{B}$ torque on the plasma which is in the counter-current direction. This return current is similar, but unrelated, to the return current that creates the 'instantaneous' torque. Note that the 'instantaneous' torque is always directed in the same direction as NBI and is unaffected by the ripple, while the TF ripple induced $\boldsymbol{j} \times \boldsymbol{B}$ torque is always directed counter-current. NBI particles that are directly injected in the TF ripple should be treated similar to those trapped in banana-orbits and these would therefore contribute to the 'instantaneous' torque. This contribution may, however, be negligible compared with the counter-current torque generated by the losses of these particles.

For high enough TF ripple the torque from the non-ambipolar radial current, here called $T_{\mathrm{RIP}}$, can become non-negligible and have an important effect on the plasma rotation. The reduction of plasma rotation or angular momentum with TF ripple, as observed in figure 1, implies a loss of momentum compared with that injected by the NB system. The momentum loss from the plasma is, however, transferred via the TF ripple to the coils, thus momentum conservation is not violated.

The first mechanism, involving friction with particles trapped in the TF ripple, tends to relax the plasma rotation to zero, while the second mechanism, related to bananaorbit diffusion, actually drives rotation in the counter-current direction. This first effect depends on the number of particles trapped in the TF ripple, while the second will be stronger in the case of a larger fraction of particles that are trapped in banana-orbits. The latter effect can be illustrated by comparing discharges with a large TF ripple but using different angles of NBI. At JET the NB system can apply near tangential injection with a tangency radius of $R_{\mathrm{T}}=1.85 \mathrm{~m}\left(\theta_{\text {inject }} \sim 25^{\circ}\right)$ and so-called normal injection with smaller tangency radius of $R_{\mathrm{T}}=1.31 \mathrm{~m}\left(\theta_{\text {inject }} \sim 17^{\circ}\right)$ (see figure 2$)$. The major radius at JET is $R_{\mathrm{O}}=2.95 \mathrm{~m}$. The beam energy varies between 70 and $130 \mathrm{keV}$. In figure 3 , two discharges are shown both with a TF ripple of $\delta=0.5 \%$ but one with predominantly tangential and the other so-called normal NBI. The powers in both discharges are chosen such that the total toroidal torque applied by $\mathrm{NBI}\left(T_{\mathrm{NBI}}=15.1 \pm 1 \mathrm{Nm}\right)$ is the same in both discharges. The overall rotation of these two discharges is reduced considerably compared with similar discharges with minimum ripple $(\delta=0.08 \%)$. Furthermore, it can be seen that the plasma with tangential NBI has significantly larger (20\%) angular momentum compared with the one with normal 


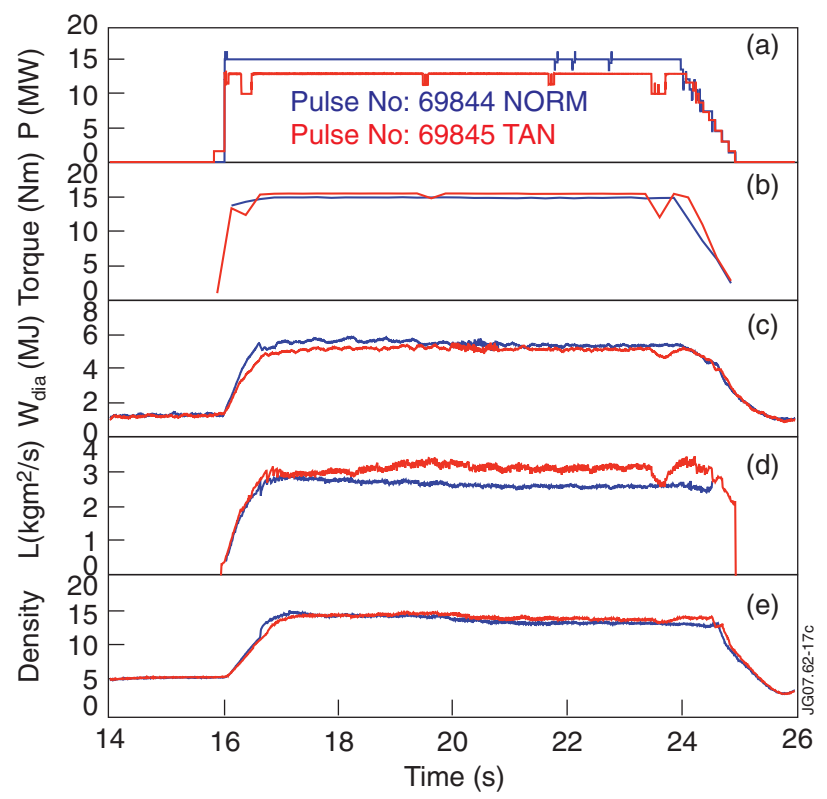

Figure 3. (a) NBI power for the two JET discharges with predominantly normal and tangential injection in blue and red, respectively. Lower power was injected in the discharge with tangential NBI. The TF ripple in both discharges was $\delta=0.5 \%$. (b) The toroidal torque applied by NBI. (c) The diamagnetic energy. (d) The total angular momentum. The lower angular momentum is found for the discharge with normal NBI. $(e)$ The line-integrated density in $10^{19} \mathrm{~m}^{-2}$.

injection. Such a difference is not observed in discharges with a standard JET ripple of only $\delta=0.08 \%$, indicating that the difference is due to TF ripple.

The observed difference in toroidal rotation can be explained by the fact that normal NBI produces more ions that are trapped in banana-orbits, which are affected by the TF ripple. The higher density of trapped particles in the presence of a large TF ripple results in a larger counter torque induced by the radial banana-orbit diffusion ( $\left.T_{\mathrm{RIP}}\right)$, hence reducing the rotation. ASCOT indeed calculates a larger power loss fraction for the case with normal bank injection, $13 \%$ compared with $9 \%$. ASCOT is able to calculate the resulting induced $\boldsymbol{j} \times \boldsymbol{B}$ torque due to these losses. It determined that these extra losses resulted in a lower torque (by $2.7 \mathrm{Nm}$ ) for plasma with normal NB. The total torques on the plasma calculated by ASCOT, including the external NBI $\left(T_{\mathrm{NBI}}\right)$ and that induced by $\mathrm{TF}$ ripple losses $\left(T_{\mathrm{RIP}}\right)$, were $T_{\mathrm{NBI}}+T_{\mathrm{RIP}}=T_{\mathrm{ASCOT}}=9.1 \mathrm{Nm}$ and $T_{\mathrm{ASCOT}}=11.8 \mathrm{Nm}$ for the normal and tangential cases, respectively.

The dependence on the fraction of particles trapped in banana-orbits indicates that the toroidal rotation is likely affected by the banana-orbit diffusion losses. ASCOT calculations show that the fraction of particles lost due to trapping by the TF ripple is negligible (less than $0.1 \%$ ) for the small to medium levels of TF ripple discussed in this paper $\delta<1.0 \%$. Hence, the friction of these trapped particles with the rotating plasma can be neglected. The ASCOT calculations have shown that the radial profile of the TF ripple induced torque is not confined to the edge of the plasma. The radial profile of the TF ripple driven $\boldsymbol{j} \times \boldsymbol{B}$ torque can affect the region from $\rho>0.5$ for $\delta=1 \%[8]$.

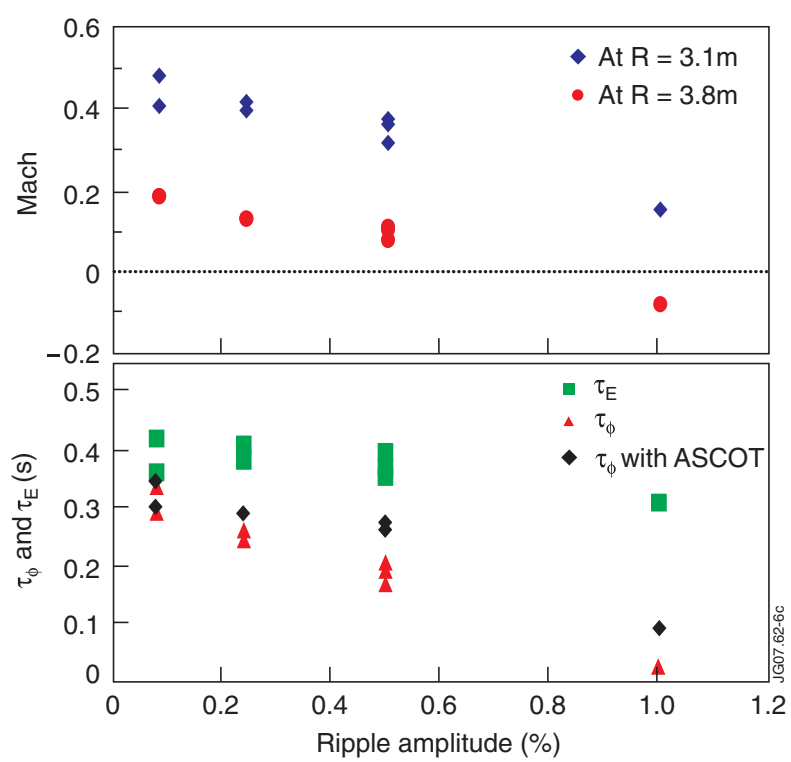

Figure 4. Series of identical type I ELMy H-modes pulse but with increasing TF ripple. The top graph shows again the core $(R=3.1 \mathrm{~m}, \rho \sim 0)$ and edge $(R=3.8 \mathrm{~m}, \rho \sim 0.9)$ Mach number, while the bottom graph presents the energy (green squares) and momentum (red triangles) confinement times. The black diamonds show the momentum confinement times with the toroidal torque calculated by ASCOT.

\section{Comparison of experiment and modelling}

In figure 4 a dedicated TF ripple scan is shown, where besides the TF ripple, the other plasma parameters are kept unchanged in a series of ELMy H-mode discharges. Again the Mach number and total angular momentum are found to decrease with TF ripple amplitude. Furthermore the momentum confinement time, $\tau_{\phi}$, defined as the ratio of the total angular momentum and the externally applied torque by $\mathrm{NBI}\left(T_{\mathrm{NBI}}\right)$, is found to be much smaller than that of the energy confinement time. In JET for a standard TF ripple $(\delta=0.08 \%)$ these parameters are found to be of the same order of magnitude [12]. The slight drop in energy confinement time with TF ripple is due to the degradation of the $\mathrm{H}$-mode pedestal for larger $\mathrm{TF}$ ripple as discussed in detail in [13].

As shown in the previous section, the torque on the plasma is not due to NBI alone but also TF ripple induced losses also play a role. Thus, the momentum confinement time should be determined using the torque as calculated by ASCOT, which include TF ripple effects $\left(T_{\mathrm{ASCOT}}=T_{\mathrm{NBI}}+T_{\mathrm{RIP}}\right)$. These values are shown in figure 4 to be larger than the original confinement times but the values still drop with ripple amplitude and are considerably lower than those of the energy confinement. Figure 5 shows that the angular momentum for these discharges scales with the torque as calculated by ASCOT. All these discharges had approximately the same NBI torque $\left(T_{\mathrm{NBI}}\right)$. The offset scaling indicates that either the momentum transport is strongly affected by the TF ripple or the TF ripple induced counter torque $\left(T_{\mathrm{RIP}}\right)$ as calculated by ASCOT is too small. Negative or counter rotation has been observed at the edge in discharges for which ASCOT still calculates a positive total torque. The ASCOT calculations show that for a TF ripple $\delta=1 \%$ approximately $67 \%$ of the co-NBI torque $\left(T_{\mathrm{NBI}}\right)$ is lost 


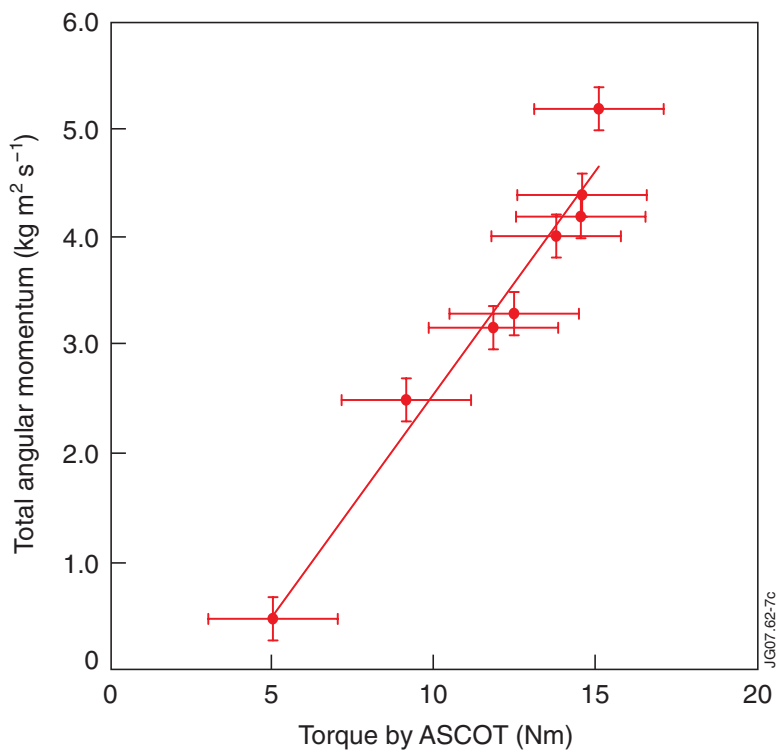

Figure 5. The total angular momentum as a function of the torque as calculated by ASCOT (including TF ripple effects) for the discharges shown in figure 4 . The NBI torque for each of these discharges varied between $T_{\mathrm{NBI}}=14.8-17.1 \mathrm{Nm}$. The main variation of total torque as calculated by ASCOT was obtained by changing the TF ripple amplitude.

due to a counter acting TF ripple torque $\left(T_{\mathrm{RIP}}\right)$. However, the negligible angular momentum indicates that this may be nearer to $100 \%$ of the NBI torque $\left(T_{\mathrm{NBI}} \sim-T_{\mathrm{RIP}}\right)$ or that another source for negative torque is present. If one would assume the same momentum confinement time for all the discharges in figure 5, one could determine the required torque. For the discharge with almost negligible angular momentum $(\delta=1 \%)$ this gives $\sim 2 \mathrm{Nm}$, which is several $\mathrm{Nm}$ smaller than provided by the ASCOT $(\sim 5 \mathrm{Nm})$. The effect of TF ripple on the torque is much stronger than on the fractional loss of heating power, which is estimated to be in the order of about $18 \%$ in this case. A further increase of TF ripple would have led to a spin-up in the counter direction.

The edge counter-current rotation with $\mathrm{TF}$ ripple amplitude of $\delta=1 \%$ seems similar to that observed in JT-60U prior to the installation of ferrite inserts when the $\mathrm{TF}$ ripple was in the order of $\delta=1-2 \%$. The countercurrent rotation in JET was found to depend on local plasma conditions. For example, a fast increase of counter-current rotation was seen at times when an H-mode pedestal built up. Furthermore, increasing the edge density could reduce the counter rotation. In figure 6 , the edge $(R=3.8 \mathrm{~m}$, $\rho \sim 0.9)$ and core $(R=3.1 \mathrm{~m}, \rho \sim 0)$ rotation for a series of four discharges (ELMy H-mode) is shown with varying amounts of gas dosing. The edge temperature was reduced while the edge density was increased due to the gas dosing, yielding a change in normalized edge collisionality from $v^{*}=$ 0.08 to 0.28 . Levels up to $4.5 \times 10^{22}$ electrons per second were used. The fast counter-current rotation at the edge of $M_{\text {edge }}=-0.06$ for the unfuelled case reverses to the co-current direction at high levels of gas dosing. Consequently, the angular momentum increases. Although there are variations in density and temperature, these are mainly found at the edge, and do not significantly alter the ASCOT calculations

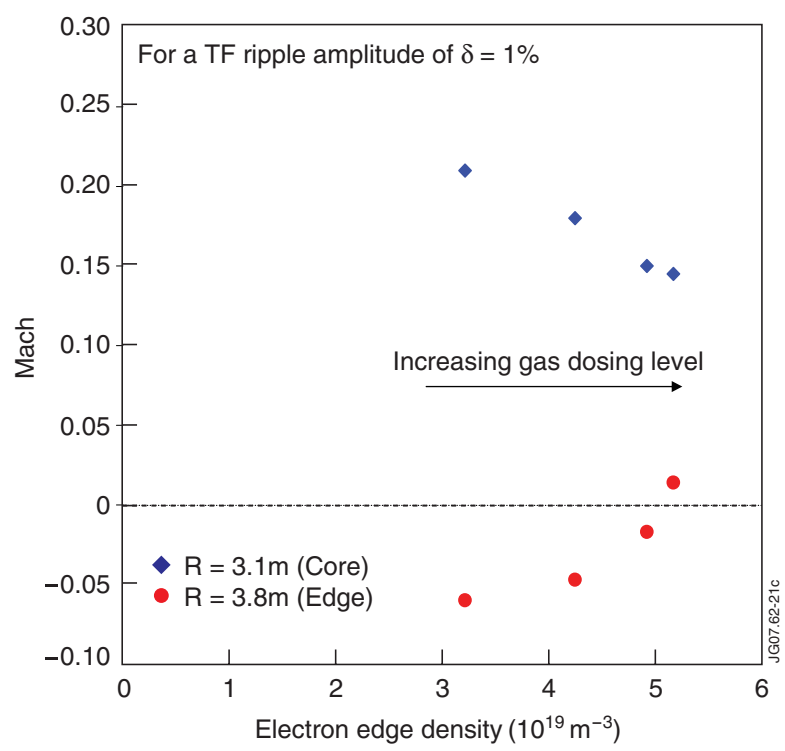

Figure 6. The core (blue diamonds) and edge (red circles) plasma rotation as a function of the edge density (e.g. increasing gas dosing).

for this series, all of which produce similar values of effective torque. The total angular momentum for the four discharges increases with density (from $L_{\text {tot }}=0.98$ to $1.20 \mathrm{~kg} \mathrm{~m}^{2} \mathrm{~s}^{-1}$ ). This increase was mainly due to the lower counter rotation at the edge. The energy confinement time was, however, similar for all the four discharges. The change in angular momentum suggests that either the momentum confinement improves significantly with edge density or that the torque differs from that calculated by ASCOT. The actual torque may be affected by the higher edge density.

During the TF ripple experiments, the fastest cases of counter-current rotation have been observed in advanced tokamak scenarios. Compared with the base-line ELMy $\mathrm{H}$-mode scenario these usually have high edge temperatures $(\sim 1 \mathrm{keV})$. In advanced tokamak scenarios predominantly heated with co-current NBI and a TF ripple of $\delta=1 \%$, a large area of the plasma between $\rho=0.6$ and 1 was found to rotate in the counter-current direction with co-rotation in the core [14].

These observations suggested that the level of negative, counter-current, edge rotation in the presence of large $\mathrm{TF}$ ripple would depend on the local temperature. In figure 7, the measured core and edge Mach numbers are shown for JET plasmas with a TF ripple of $\delta=1 \%$. All these discharges are predominantly heated by co-NBI. The edge rotation clearly correlates with the temperature. Discharges with very high edge temperatures produce a stronger counter-current rotation in the presence of a large TF ripple. The normalized edge collisionality ranged from $v^{*}=0.04$ for the high temperature cases to $v^{*}=0.5$ for the lower temperature and high density cases. This trend can partly explain the spread in the edge Mach numbers observed in figure 1 , at the specific ripple amplitude of $\delta=1 \%$.

\section{Discussion}

TF ripple induced particle losses have been shown to affect the toroidal rotation of the plasma. The basic mechanisms 


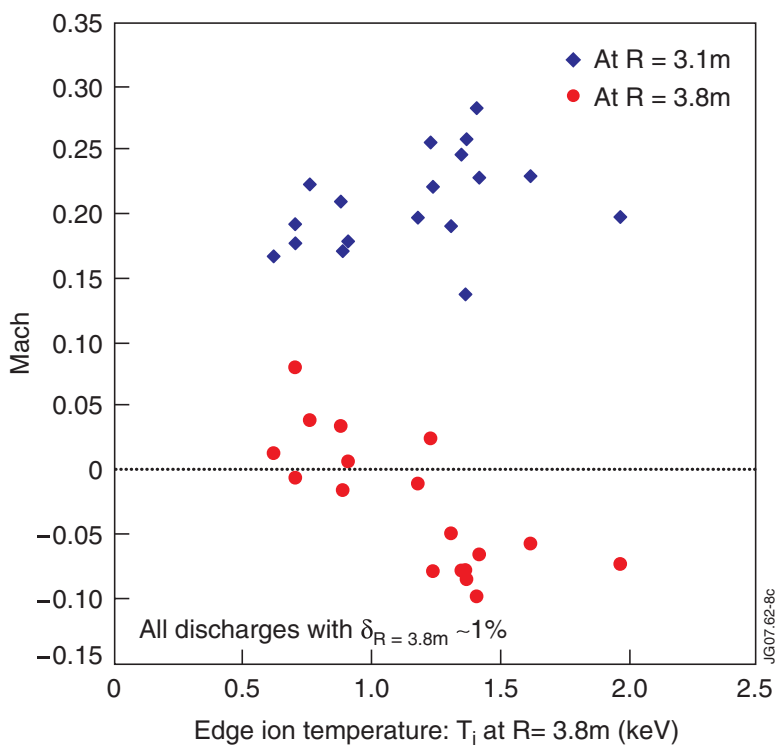

Figure 7. The centre and edge Mach number $(R=3.8 \mathrm{~m}, \rho \sim 0.9)$ for all JET discharges with a TF ripple of $\delta \sim 1 \%$ (at $R=3.8 \mathrm{~m}$ ) as a function of the local ion temperature.

behind the effect and experimental characteristics have been described.

Friction with particles trapped in the TF ripple cannot explain counter rotation. Furthermore, ASCOT calculations have shown that the loss fraction of particles due to trapping in the TF ripple is negligible (less than $0.1 \%$ ) for the levels of TF ripple discussed in this paper, $\delta<1.0 \%$. The dominant mechanism that drives the observed edge counter rotation in JET discharges with a large TF ripple $\delta>0.5 \%$ can be associated with banana-orbit diffusion of trapped energetic ions. However, calculations of the induced torque due to these losses do not fully explain the observations. The edge toroidal rotation in the presence of a large TF ripple in JET depended on the local ion temperature or possibly collisionality, suggesting that other ion losses, possibly those of thermal ions, may be involved [15]. At a TF ripple amplitude of $\delta=1 \%$ these additional losses are thought to be responsible for a (counter) torque of several $\mathrm{Nm}$. The effect of TF ripple on thermal ions has so far not been included in the ASCOT calculations shown in this paper.

Even for small TF ripple amplitudes of $\delta \sim 0.5 \%$, which is expected in ITER, the JET plasma rotation was significantly reduced compared with normal levels. In JET discharges with a TF ripple of $\delta \sim 0.5 \%$ the counter-current torque was found to be in the order of $20-30 \%$ of that supplied by the JET NBI system in the co-current direction. It should be noted that TF ripple in general does not reduce rotation, it merely provides a counter-current torque. In JT-60U with almost perpendicular NBI and larger ripple $\delta \sim 1 \%$ the ripple induced ion losses may have been the dominant drive for the observed counter-current plasma rotation [5]. Furthermore, a larger angular momentum was observed when using counter-current compared with the co-current NBI in the presence of a TF ripple of $\delta \sim 0.5 \%$ [7]. With counter-current NBI the TF ripple induced torque actually supports the NBI torque and enhances the angular momentum, albeit in the counter direction. This shows that the orientation of the NBI, e.g. tangential versus normal/perpendicular injection, at these ripple amplitudes is of importance.

Predictions for plasma rotation in ITER often assume that the momentum and energy confinement times are proportional and do not include TF ripple effects [16]. Predicting plasma rotation in ITER by extrapolating from present devices, such as JET, which have significantly lower TF ripple may, however, be more complicated. The TF ripple induced torque is always in the counter-current direction and will reduce the NBI co-current torque, yielding a lower rotation in ITER than presently expected. These experiments have shown that for TF ripple amplitudes of $\delta \sim 0.5 \%$ the Mach numbers are approximately $30 \%$ low. However, to accurately quantify these effects detailed modelling of TF ripple effects on ITER rotation is required. The effect of thermal ion losses on the torque is being investigated, by modelling these losses with ASCOT. Initial results indicate that these losses may indeed contribute to the counter-current torque on the plasma [8].

\section{Acknowledgments}

This research was funded partly by the United Kingdom Engineering and Physical Sciences Research Council and by the European Communities under the contract of Association between EURATOM and UKAEA. The views and opinions expressed herein do not necessarily reflect those of the European Commission. This work was carried out within the framework of the European Fusion Development Agreement.

\section{References}

[1] Tobita K. et al 1995 Nucl. Fusion 351585

[2] Redi M.H. et al 1995 Nucl. Fusion 351509

[3] JET TEAM 1993 Proc. 14th Int. Conf. on Plasma Physics and Controlled Nuclear Fusion (Würzburg, Germany, 1992) (Vienna: IAEA) vol 1 p 429

[4] Tubbing B. et al 1995 Proc. 22th EPS Conf. on Plasma Physics Controlled Fusion (Montpellier, France, 1995) vol 19C p IV-001

[5] Koide Y. et al 1993 Plasma Phys. Control. Nucl. Fusion Res. 1 777

[6] Yoshida M. et al 2006 Plasma Phys. Control. Fusion 481673

[7] Urano H. et al 2007 Nucl. Fusion 47706

[8] Salmi A. et al 2007 ASCOT modelling of ripple effects on plasma rotation Proc. 11th Int. Workshop on Plasma Edge Theory in Fusion Devices (Takayama, Japan, 2007)

[9] de Vries P.C. et al 2006 Plasma Phys. Control. Fusion 481693

[10] Zastrow K.-D. et al 1998 Nucl. Fusion 38257

[11] Goldston R.J., White R.B. and Boozer A.H. 1981 Phys. Rev. Lett. 47647

[12] Mikhailovskii A.B. 1995 Phys. Lett. A 198131

[13] Saibene G. et al 2007 Toroidal field ripple effects on H-modes in JET and implications for ITER Proc. 34th EPS Conf. on Plasma Physics Controlled Fusion (Warsaw, Poland, 2007) http://www.eps2007.ifpilm.waw.pl/pdf/O4_001.pdf

[14] de Vries P.C. et al 2007 Toroidal field ripple and the formation of internal transport barriers Proc. 34th EPS Conf. on Plasma Physics Controlled Fusion (Warsaw, Poland, 2007) http://www.eps2007.ifpilm.waw.pl/pdf/O4_002.pdf

[15] Parail V. et al 2006 Proc. 21st Int. Conf. on Fusion Energy 2006 (Chengdu, China, 2006) (Vienna: IAEA) CD-ROM file TH/P8-5

[16] Polevoi A.R. et al 2002 Proc. 19th Int. Conf. on Fusion Energy 2002 (Lyon, France, 2002) (Vienna: IAEA) CD-ROM file CT/P-08 